\title{
Claudia Lux baut Nationalbibliothek in Katar auf
}

Zusammenfassung: Das Emirat Katar investiert viel in Bildung und Forschung und legt einen besonderen Schwerpunkt auf die Entwicklung der Informationsgesellschaft und somit auch auf den Aufbau einer modernen Nationalbibliothek. Die frühere Generaldirektorin der Zentral- und Landesbibliothek Berlin (ZLB), Claudia Lux, wechselte im April 2012 nach Katar und baut dort eine Nationalbibliothek mit Universitätsbibliothek und Metropolenbibliothek auf, die im Jahr 2015 eröffnen soll. Das vorliegende Interview ${ }^{1}$ beschreibt die Entwicklungsschritte, die in diesem Prozess bereits erfolgt sind und die Herausforderung, die dieses Projekt darstellt.

Schlüsselwörter: Katar, Nationalbibliothek, Neubau, Neustrukturierung

\section{Claudia Lux sets up national library in Qatar}

Abstract: The Emirate of Qatar invests much into education and research paying attention in particular to the development of the knowledge society and thus to setting up a modern national library. The former director general of "Zentral- und Landesbibliothek Berlin” (ZLB), Claudia Lux, left her position there for a new post in Qatar. There she is responsible for setting up a national library housing both a university and a metropolitan library and which is due to open in 2015. The interview presented here describes the steps already taken in the course of the process and the challenges involved.

Keywords: Qatar, national library, new construction, reorganization

Prof. Dr. Claudia Lux: E-Mail: clux@qf.org.qa

Das Emirat Katar investiert sehr viel in Bildung und Forschung, um seine Vision 2030 von einem wirtschaftlich diversifizierten Land zu erreichen. Ein Ziel dabei ist die Entwicklung der Informationsgesellschaft und somit der Aufbau einer

1 Der Bibliotheksdienst plant, neben Aufsätzen und Notizen zukünftig auch Interviews zu interessanten Themen zu veröffentlichen. 
modernen Nationalbibliothek. Es sicherte sich dafür deutsches Know-how, denn die Generaldirektorin der Zentral- und Landesbibliothek Berlin (ZLB), Claudia Lux, wechselte im April 2012 nach Katar. Claudia Lux baut dort eine Nationalbibliothek mit Universitätsbibliothek und Metropolenbibliothek auf, die im Jahr 2015 eröffnen soll.

\section{Bibliotheksdienst: Ihr Weggang aus der ZLB hat die meisten Beschäftigten und die Fachwelt überrascht. Was waren Ihre Beweggründe für diesen Schritt?}

Claudia Lux: Ich muss gestehen, dass ich mich im Oktober 2010, als die erste Anfrage aus Katar kam, nicht gleich angesprochen fühlte, weil ich mir gar nicht vorstellen konnte, aus Berlin und von der ZLB wegzugehen. Als aber dann die Anfragen aus Doha intensiver wurden und ich das Projekt in Katar im März 2011 selbst kennenlernen konnte, hat es mich doch gepackt. Die Chance, in Katar einen Bibliotheksbau etwas schneller als in Berlin realisieren zu können, war besonders reizvoll.

Ich habe immer sehr gerne in der ZLB gearbeitet. Der Abschied war deshalb für mich nicht leicht. Allerdings waren das Angebot und die Chancen in Katar so attraktiv, dass ich das Abenteuer einfach wagen wollte, bevor mich das Greisenalter erwischt. Vor allem nachdem die Entscheidung zum Bau der ZLB gefallen war und der Fertigstellungstermin auf zehn Jahre kalkuliert wurde, ein Zeitpunkt weit außerhalb meiner beruflichen Laufbahn. Auch wenn ich wirklich intensiv und hart für die Entscheidung gearbeitet und alle wichtigen Personen vorher kontaktiert und bearbeitet hatte, konnte man ja bis zuletzt nicht sicher sein, dass der neue Berliner Senat mit der großen Koalition den Beschluss zum Neubau der ZLB im Herbst 2012 wirklich fassen würde. Für mich war das ein wichtiger Schritt, der mir die Entscheidung für Katar sehr erleichtert hat, da damit das Bauprojekt ZLB auf den Weg gebracht war. Nun müssen andere den Ball im Spiel halten und für die Realisierung sorgen.

\section{Das Emirat Katar hat andere politische und gesellschaftliche Verhältnisse, als wir sie aus Europa kennen. Wie sind für Sie als Frau die Arbeits- und Lebensbedingungen in Doha?}

Als Frau habe ich hier in Katar, mit einer Bevölkerung von 85 \% Ausländern, keine Probleme. Auto fahren, Restaurants besuchen, spazieren gehen - alles kann man hier als Frau auch alleine machen und wird respektiert. In der Kultur der Golfstaaten gibt es eine starke Trennung von Frauen und Männern bei familiären Festen und religiösen Treffen, allerdings kaum bei öffentlichen Veranstaltungen. Wir haben einige Kolleginnen, die hier alleine leben und sich sehr wohl fühlen, während ich ja mit meinem Mann nach Katar ziehen konnte. 
In der Zeitung verfolgen wir die Diskussion um Veränderungen, z. B. um ein neues Arbeitsrecht, Rechte der Frauen usw. Neulich gab es einen Artikel, in dem die Frauen $50 \%$ aller Leitungspositionen einforderten. Davon ist man - genau wie bei uns - noch weit entfernt, aber an verschiedenen Stellen sitzen Frauen am Hebel. Die junge Chefin des Museumskonzerns hier in Katar wurde neulich als einflussreichste Frau in der internationalen Kunstszene bezeichnet.

Das ganze Land ist eine Baustelle mit riesigen Infrastrukturmaßnahmen, Straßen, Eisenbahn, U-Bahn, Hafen, Abwasserkanäle werden gleichzeitig gebaut. Kilometerlange Bauzäune zeigen, was kommt: Gigantische Einkaufszentren und neue Museen, Forschungseinrichtungen und die Nationalbibliothek sind im Bau. Mit den Stadien für die Fußballweltmeisterschaft 2022 wurde noch nicht richtig begonnen, nur an einem Stadion wird schon gebaut. Fußball ist hier Nationalsport und die ebenfalls neue Trainerin der katarischen Mädchenfußballmannschaft ist eine Deutsche. Im Emirat werden die juristischen und politischen Regelungen von Grund auf schrittweise erneuert, immer in vorsichtiger Abstimmung mit den verschiedenen gesellschaftlichen Gruppen, um möglichst alle mitzunehmen.

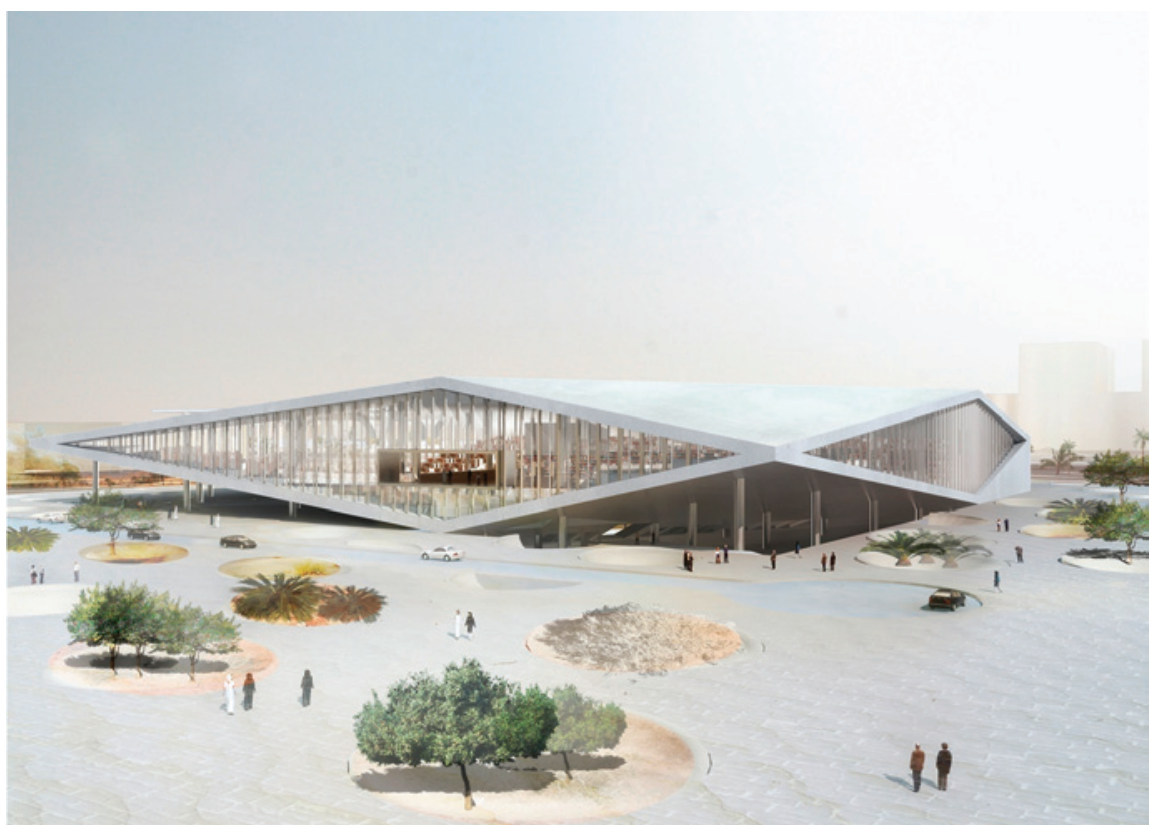

Abb. 1: Außenansicht neue Nationalbibliothek in Doha. 
In den letzten zwei Jahren habe ich viel von der besonderen Kultur Katars erfahren können. Man ist hier sehr um Konsens in den eigenen Reihen bemüht und es wird sehr viel Rücksicht auf geäußerte Interessen genommen. Die Stiftung hat klare Regeln niedergelegt und schult Mitarbeiter und Führungskräfte intensiv, damit diese Regeln, die sich an internationalen Standards für Wirtschaftsorganisationen orientieren, auch eingehalten werden. Den Bereich der privaten Wirtschaft kenne ich zu wenig.

Einige Frauen sind außergewöhnlich erfolgreich im Beruf. Unsere neue Bibliothek, bei der die Außenhülle nahezu fertig ist und nun der Innenausbau stark vorangetrieben wird, hat zwei starke katarische Frauen als Bauleiterinnen, die direkt mit den Projektleitern und Baufirmen verhandeln. Und auf unserer Baustelle, die ich nur mit Helm und Sicherheitsschuhen betreten darf, ist der Ton immer ruhig und nie laut. Auch wenn wir gemeinsam mit dem Architekten Rem Koolhaas zusammensitzen, geht es immer darum, Konsens $\mathrm{zu}$ finden, wobei unser Stararchitekt genial mit seinen Lösungen und Kompromissen ist.

Von meinen 51 Mitarbeitern und Mitarbeiterinnen sind gegenwärtig 13 katarisch. Sie alle tragen ihre katarische Kleidung aus kulturellen, nicht aus religiösen Gründen, Männer das weiße und Frauen das schwarze Gewand. Die anderen Mitarbeiter und Mitarbeiterinnen sind aus verschiedenen arabischen Ländern, aus den Philippinen, Indien, Aserbaidschan, USA, Kanada, England, Sri Lanka, Australien und tragen normale Kleidung, zwischen Jeans und Anzug mit Krawatte. Einige Ägypterinnen tragen ihr Kopftuch, eine Inderin manchmal ihr indisches Gewand. Interkulturelle Kompetenz und gegenseitiger Respekt sind Basis der Zusammenarbeit in Katar und ein Verstoß dagegen wird nicht toleriert. Wir ausländischen Arbeiter und Angestellte sind in Katar Gäste und ohne die klare Akzeptanz der Regeln des Landes und ohne eine hohe Toleranz kann es mit den sehr unterschiedlichen Nationalitäten nicht funktionieren.

\section{Wie hat sich das Projekt in den letzten zwei Jahren entwickelt?}

Hier ein paar Highlights aus den ersten beiden Jahren:

Gleich zu Beginn meiner Tätigkeit wurde schriftlich von der Vorsitzenden des Stiftungsrats der Katar Stiftung, Ihre Hoheit und Frau des damaligen Emirs Sheikha Moza bint Nasser, bekannt gegeben, dass die Bibliothek jetzt die Nationalbibliothek ist und dass ich als Projektdirektorin ihr direkt unterstellt bin. Das hat mir den Beginn natürlich sehr erleichtert. Einige angefangene Teilprojekte lagen brach und mussten wieder aufgenommen werden.

Im ersten Sommer 2012 haben wir eine Strategie für die neue Nationalbibliothek entworfen und diese gemeinsam mit den Mitarbeitern und Mitarbeiterinnen in einen Businessplan und eine Zeitplanung für die nächsten fünf Jahre umgesetzt. Die Strategie spricht davon, dass wir Nationalbibliothek, Universitätsbib- 
liothek und Metropolenbibliothek in einem sein werden - also öffentliche und wissenschaftliche Bibliothek zusammen. Wir blicken nach Singapur und nach Dänemark zu unseren Vorbildern und werden dennoch etwas völlig Neues und Eigenständiges entwickeln.

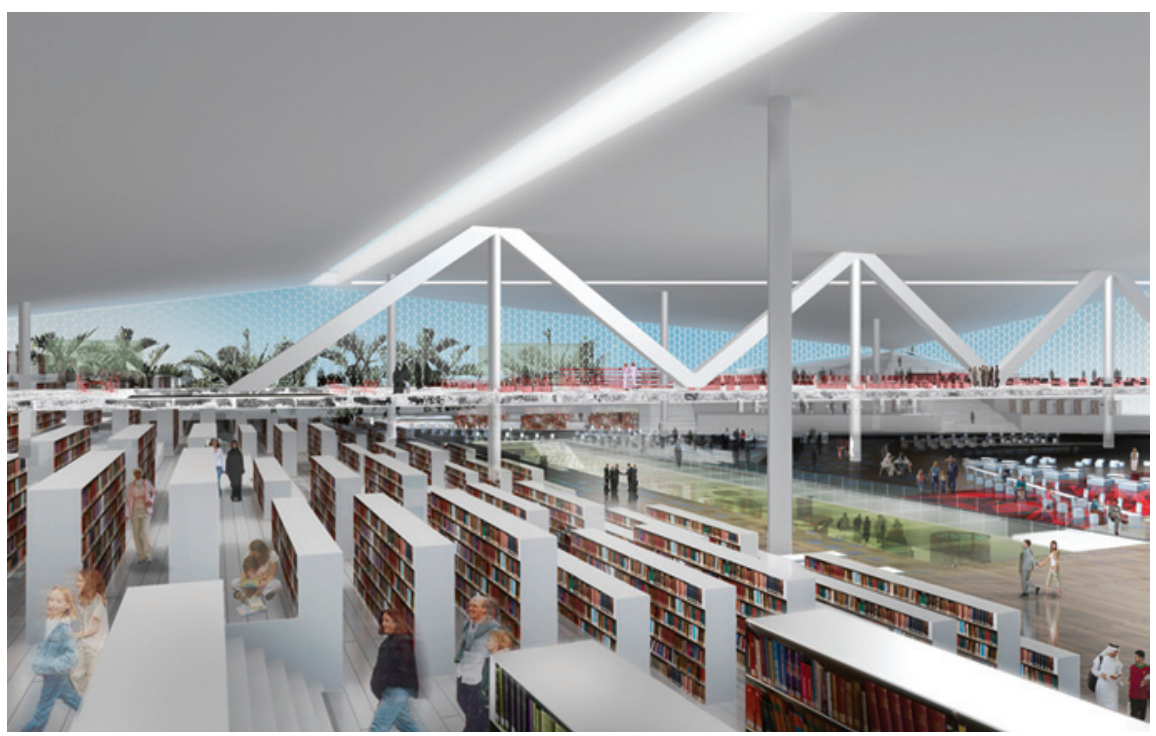

Abb. 2: Innenansicht (Entwurfsskizze) neue Nationalbibliothek.

Gleich als Erstes haben wir mit der British Library ein Projekt zur Digitalisierung von 500.000 Seiten des Indian Office Archives, das viele Dokumente zum Arabischen Golf enthält, abgeschlossen. Gleichzeitig entsteht damit ein Portal für weitere Digitalisate der Qatar National Library (QNL). Die World Digital Library unterstützen wir finanziell, damit arabische Handschriften dort aufgenommen werden. In der nationalen Digitalisierungsstrategie, die das ICT-Ministerium Anfang 2014 zur Diskussion veröffentlicht hat, ist die Nationalbibliothek als einer der wichtigsten Digitalisierungspartner für das nationale Erbe benannt worden und ein Partnerabkommen wurde mit uns unterzeichnet.

Anfang 2013 wurde unser umfassendes Konzept mit allen Planungsunterlagen positiv vom Stiftungsrat der Katar Stiftung verabschiedet. Nun bereiten wir alles vor, um die realen und virtuellen Regale der neuen Bibliothek füllen zu können. So wurde ein allgemeines Bestandsprofil erarbeitet, das jetzt in Profile für unsere Lieferanten präzisiert wird.

Wir haben noch nicht genügend Personal, sollen aber in den nächsten zwei Jahren auf über 170 Personen wachsen. Außerdem musste die Verwaltung über- 
zeugt werden, dass der Ausbau der Digitalen Bibliothek mit Datenbanken und E-Books auch schon vor der Eröffnung des Gebäudes notwendig ist. Dabei versuchen wir meist Nationallizenzen, wie für Naxos Music Library, zu beschaffen. Inzwischen haben wir über 5.000 eingetragene Kunden, die uns digital nutzen. Auch unsere Webseite konnte in den ersten Monaten online gehen: www.qnl.qa.

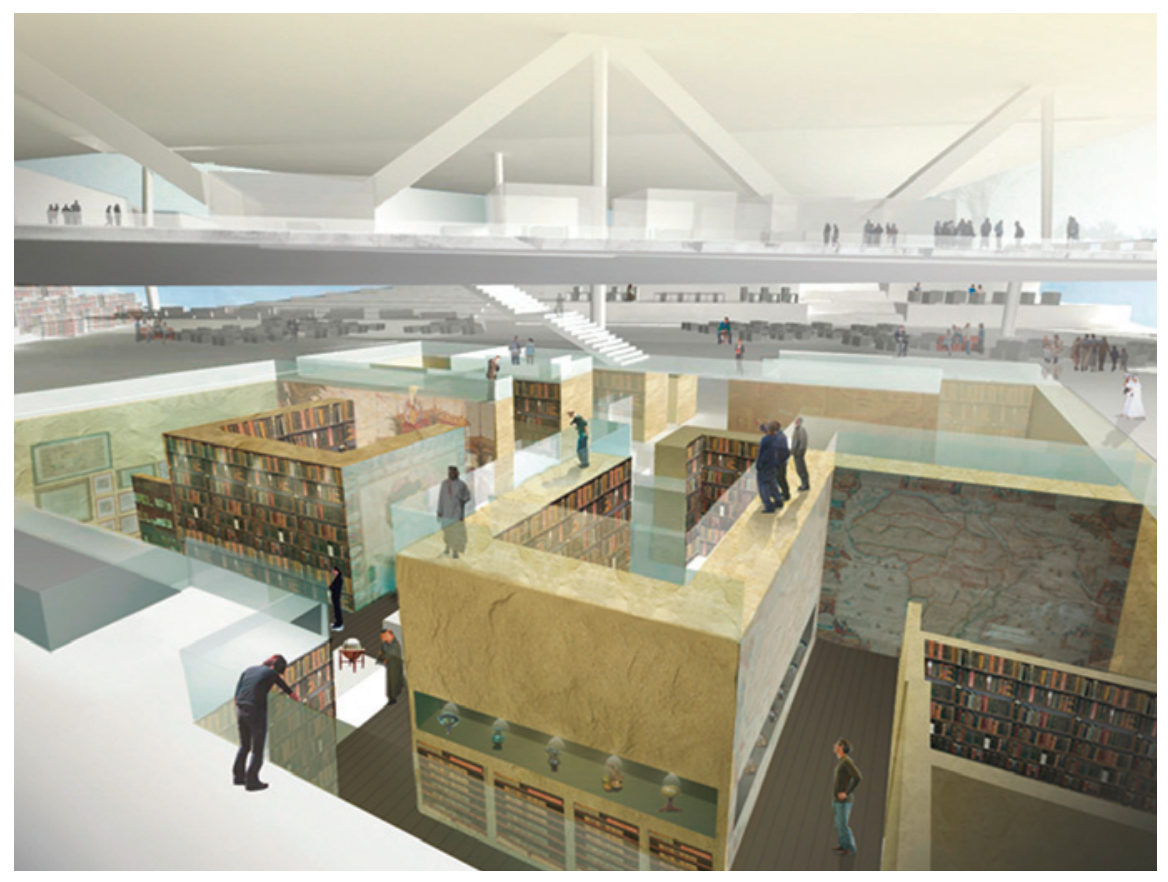

Abb. 3: Innenansicht (Entwurfsskizze) neue Nationalbibliothek.

Einige neue wertvolle Werke für unsere Historische Sammlung haben wir auf verschiedenen Auktionen erstanden. Inzwischen habe ich eine professionelle Leitung für diesen Bereich, aber bis vor wenigen Monaten musste ich mich noch selbst darum kümmern. So lernte ich einiges über die Geschichte der Golfstaaten und über das Erbe der arabischen Wissenschaften in Medizin, Astronomie, Mathematik, Chemie, Philosophie. Die Entwicklung der arabischen Wissenschaften zu einem Zeitpunkt, als bei uns das Mittelalter herrschte, hat die Entstehung der europäischen Renaissance erheblich beeinflusst.

Mit meinem Kollegen von der alten Nationalbibliothek Dar-Al-Kutub habe ich ein Buch zu 50 Jahre Nationalbibliothek Katar herausgegeben und darin einen Artikel, der die Zielstellung der neuen Bibliothek beschreibt, veröffentlicht sowie einen Vortrag dazu auf der Konferenz der Arabischen Bibliothekare AFLI, 
die 2012 Doha tagte, gehalten. Weiterhin haben wir die offizielle Ankündigung der neuen Nationalbibliothek Katars als große Veranstaltung im November 2012 gefeiert. Dabei gab es eine sehr gute Podiumsdiskussion mit der Vorsitzenden des Stiftungsrats, die klare Bekenntnisse zu einer Bibliothek mit hohem digitalem Anteil, zur Bibliothek als Treffpunkt für alle und zu Öffnungszeiten rund um die Uhr abgab.

Die Baustelle war im April 2012 nur eine Baugrube, jetzt Anfang 2014 steht dort ein recht großes attraktives Gebäude, allerdings ist noch einiges am Innenausbau zu leisten und wir rechnen mit Fertigstellung erst zum Ende dieses Jahres. Nach meiner Planung benötigen wir ein Jahr nach Übergabe des Gebäudes und werden hoffentlich Ende 2015/Anfang 2016 die Eröffnung der neuen Nationalbibliothek von Katar feiern können.

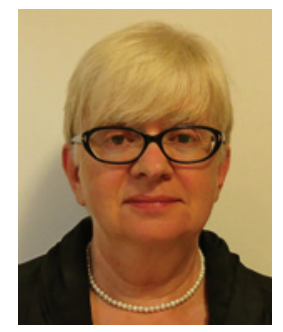

Prof. Dr. Claudia Lux

Qatar National Library

P O Box 5825

Doha, Qatar

E-Mail: clux@qf.org.qa 